\title{
Le développement durable dans la société du risque: le cas allemand
}

\section{Reiner Keller, Augsburg}

Le terme «Société du risque» est un concept clé dans l'analyse sociologique des sociétés contemporaines. L'expression «développement durable» indique un programme de transformation sociale considéré «objectivement nécessaire» et «moralement imposé» dans les débats publics, politiques et scientifiques. Comment caractériser le rapport entre les deux notions ? La discussion allemande sur le développement durable a tendance à ignorer les questions du risque. Ainsi, elle risque de ne faire que prolonger les dynamiques bien connues de la modernisation traditionnelle.

\section{Le débat sur le développement durable en RFA}

Le rapport BRUNDTLAND «Our Common Future» (1987) et la conférence de Rio (1992) ont déclenché un débat mondial sur la durabilité économique, sociale et écologique. Cette discussion se poursuit dans les pays divers de manière très différente. Il ne faut pas trop s'en étonner. C'est parce que ce débat est confronté aux contextes socioculturels et institutionnels très hétérogènes qui forment ses résonances nationales. En RFA, l'accueil de l'idée de durabilité est plutôt tardif. Vu la très courte durée historique de ce débat, toute évaluation de ses conséquences réelles risque de tomber dans le piège de la pure spéculation. De manière générale il faut bien reconnaitre que les structures de production et de consommation des sociétés capitalistes modernes continuent à produire des effets de non-durabilité dans les domaines des ressources de matière première et des nuisances. Dans ce sens, les dynamiques traditionnelles de la modernisation dominent les développements des sociétés d'aujourd'hui.

\subsection{Le débat public sur l'environnement}

Dès le début, le débat sur les questions écologiques en RFA est structuré suivant deux positions et discours opposés qui se confrontent sur la scène publique (KELLER 1998: 201). D'un côté, on vote en faveur des solutions techniques pour les problèmes écologiques en gardant le dynamisme de la croissance économique. Cette position peut être nommée «modernisation technico-écologique de la société industrielle». De l'autre côté, on demande une réorientation structurelle des processus du développement social. C'est la position d'une «restructuration politico-écologique». Les deux discours trouvent place dans les médias; ils sont dès le début représentés par des experts scientifiques, administratifs, économiques et politiques. La position de la modernisa- tion technico-écologique ne touche pas à l'autonomie de l'économie. Elle considère les problèmes de l'environnement comme des effets pervers inévitables du progrès, maîtrisables par des dispositifs technico-administratifs. La nature est une ressource inépuisable ou remplaçable. Objectivité et réalisme contre idéalisme, c'est son ordre de marche. Par contre, le discours de la restructuration politico-écologique demande une organisation sociale des processus économiques à travers la politique. Les problèmes écologiques sont définis comme problèmes de la société. La solution ne se trouve que dans un changement du modèle de développement social. Toute intervention de l'homme dans une nature considérée comme une ressource rare est chargée d'une potentialité de risque énorme. Mais les intérêts de profit économique empêchent la réalisation du «principe de responsabilité» (JoNAS 1990).

Le débat public sur l'environnement se déroule en quatre étapes, en suivant les polarités esquissées: Dès le milieu des années 60 jusqu'au début des années 70 , la protection de l'environnement est constituée comme objet d'une régulation politique. Cette période est suivie d'une discussion publique très polarisée sur les questions d'environnement dans les années 1975-1985, animée par le dynamisme énorme des nouveaux mouvements sociaux et une forte critique publique des dispositifs de la protection de l'environnement proposés par le gouvernement. Ensuite, entre 1986-1991, se déroule l'étape d'une compréhension plutôt consensuelle concernant les urgences de la politique d'environnement et ses dispositifs institutionnels concrets. Depuis, l'environnement perd son importance publique (KELLER 1995; Brand, Eder \& Poferl 1997).

À la fin des années 80, la protection de l'environnement a trouvé un ancrage énorme dans les institutions. Mais au même moment, les mass medias n'y trouvent plus beaucoup d'intérêt. Le déclin public de son importance est surtout fonction de l'événement spectaculaire de la chute du Mur et du processus de la réunification allemande. Le processus difficile de l'intégration économique des nouveaux Länder renforce les problèmes du chômage généralisé et il devient déclencheur d'une «Standortdiskussion» sur la situation de crise de toute l'économie allemande. S'attachant aux grands courants internationaux (mondialisation), les entreprises demandent des déréglementations économiques et écologiques. Le fait qu'en 1994 ANGeliKa Merkel remplace Klaus TöPfER à la tête du ministère de l'environnement est considéré comme expression pas seulement symbolique du déclin de l'importance de la protection de l'environnement pour le gouvernement de Helmut Kohl. Le «Haut Conseil pour les questions de 
l'environnement» constate en 1996 la régression continue des questions de la politique de l'environnement dans les années 90 (SACHVERSTÄNDIGENRAT FÜR UMWELTFRAGEN 1996). Il se peut que le changement du gouvernement en 1998 - maintenant SPD/Les Verts soit le début d'un nouvel élan dans ce domaine.

\subsection{L'accueil du «développement durable»}

Aussi bien sur le plan national que sur le plan local, l'Allemagne hésite devant la notion de durabilité. Dans le contexte de la discussion sur l'environnement, cette hésitation se fonde sur trois raisons: Premièrement, l'idée directrice de la durabilité arrive devant un public qui a accepté la légitimité de la protection de l'environnement et qui en a installé des dispostifs concrets depuis un bon moment déjà. Deuxièment, la réunification, le chômage généralisé et le débat sur la puissance menacée de l'économie allemande refoulent l'environnement de l'agenda public. Et troisièmement, les grandes associations écologiques ne voient pas d'avantages de mobilisation dans le concept de durabilité. «Et alors ?» est donc la réaction qui reflète aussi bien la fatigue sur les questions «vertes» et une dose de bonne conscience sur ce qu'on en a déjà réalisé. Cette réaction est renforcée par le gouvernement fédéral qui va très vite fixer l'étiquette de «durabilité» sur toutes ses mesures de protection de l'environnement (p.ex. le décret emballage en 1991). Ainsi la vraie importance et les nécessités d'action introduites par la notion de durabilité sont situées sur la scène internationale - les problèmes mondiaux (climat) et le développement du tiers monde. Au début, la responsabilité pour les questions de durabilité est intégrée dans le service des relations internationales du Ministère de l'environnement.

Sur l'échelle nationale, le gouvernement comprend toute sa politique de l'environnement sous l'étiquette de durabilité. En 1994, la responsabilité sociale pour la protection des fondements naturels de l'existence des générations futures devient objectif d'Etat et entre dans l'article 20a de la Constitution. Les entreprises adaptent très vite l'idée dans leur rhétorique et projettent leurs actions comme une contribution globale au développement durable (p.ex. dans les publicités de l'industrie chimique). Dans plusieurs études directrices qui rencontrent un certain écho dans le public, une multitude d'experts politiques, administratifs et scientifiques essaient de préciser la notion. On peut noter par exemple les enquêtes de l'Office Fédéral de l'Environnement (Umweltbundesamt), du Haut Conseil pour les Questions de l'Environnement (Sachverständigenrat für Umweltfragen) ou des Commissions de l'Assemblée Nationale (Enquete-Kommissionen des Deutschen

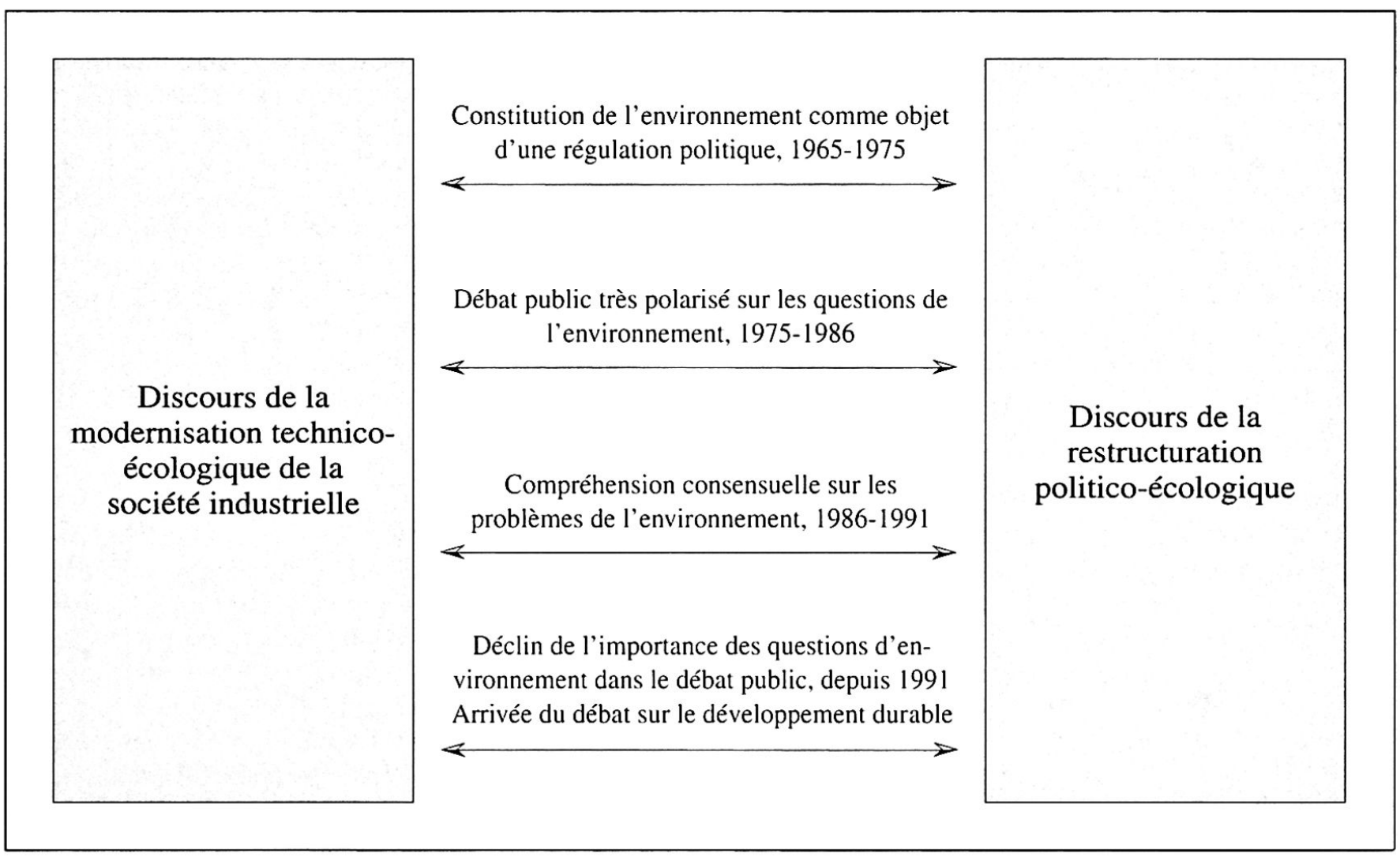

Tab. 1: Les étapes et structures du débat public sur l'environnement en RFA Etappen und Strukturen der öffentlichen Umweltdiskussion in der Bundesrepublik Deutschland Periods and structures of public discussion on environmental problems in Germany 
Bundestages). Déjà en 1992, l'association catholique d'aide aux tiers monde MisEREOR et l'association écologique BUND demandent au Wuppertal-Institut für Klima, Umwelt, Energie de travailler sur les conditions et stratégies d'une Allemagne durable. L'étude paraît en 1996 sous le titre «Zukunftsfähiges Deutschland» et attire un grand public (BUND \& MISEREOR 1996).

Bien sûr, il y a des adaptions du développement durable au niveau des Länder et sur le plan régional - cf. en Bavière à travers la promotion financière des technologies propres - mais leur impact reste très limité. La réception à l'échelle locale sous forme des processus $\mathrm{d}$ ' Agenda 21 est plus importante. Elle aussi était plutôt tardive. Les raisons pour l'hésitation vis-à-vis de cet agenda local se trouvent aussi bien dans l'interprétation internationale de l'idée de durabilité mentionnée cidessus que dans le degré fort d'institutionalisation de la protection de l'environnement dans les villes et communes. Qui plus est, l'instrument de planification urbaine intégrée avait déjà visé la coordination des aspects différents du développement municipal (Hesse 1997; KuHN \& KURTE 1997). Mais dans le courant international des processus d'Agenda 21 et avec l'amplification du débat national sur la durabilité, les projets d'Agenda local se développent dès le milieu des années 90 (cf. les cas-modèles de Berlin-Köpenick, Bremen et München). Il y a une différence très importante entre ces processus et les mobilisations écologiques d'autrefois: ce ne sont plus les nouveaux mouvements sociaux classiques, mais les institutions existantes qui en jouent le rôle initiateur. Ce sont l'administration municipale, l'Eglise ou l'université populaire, bien sûr devenues lieux de travail pour beaucoup d'anciens militants, qui organisent les activités. La pluralité organisatrice des processus d'Agenda 21 est énorme, et c'est actuellement l'échelle la plus vivante et innovatrice en matière de durabilité. Une des formes typiques d'action, ce sont des rassemblements et débats organisés par des citoyens qui travaillent sur l'adaption locale de l'Agenda. Ainsi naissent des projets modèles (p.ex. 21 foyers pour le $21^{\mathrm{e}}$ siècle; Habiter sans voiture; Isolement avancé pour les bâtiments). Alors que le nombre des participants et la résonance publique de telles activités sont plutôt modestes, elles arrivent quand même à intégrer une grand part d'acteurs locaux, soit administratifs, politiques, religieux, écologiques etc. Seuls les représentants de l'économie n'y participent pas beaucoup.

Il est bien difficile de juger des effets réels de tels processus. Du côté positif, il faut constater que les rencontres organisées mettent en contact des acteurs locaux qui s'ignoraient avant. Qui plus est, les discussions incitent à des mesures concrètes de la protection de l'environnement. Le plus prometteur se montre à travers le développement des bureaux indépendants de conseil sur l'Agenda 21 («Agenda-Agenturen») qui - en suivant le modèle des agences existantes de l'énergie - vendent leur savoir professionel sur les possibilités concrètes écologiques à tout un chacun qui paie. Mais on peut bien aussi en tirer un bilan négatif de l'Agenda 21: Les décisions prises aux cours des débats, et les buts d'actions qui en ressortent, ne semblent rester - sous les conditions existantes de toute action municipale - que des lettres au Père Noèl, qui n'entrent que très rarement dans les affaires quotidiennes des municipalités. Tout cela ne veut pas dire que les villes et communes n'accordent pas d'importance à la protection de l'environnement.

\subsection{Chances et risques d'une idée directrice}

Le concept de «développement durable» est compris surtout comme idéal abstrait, idée régulatrice ou directrice. Sans doute, il y a un vaste consensus là-dessus. Quelques-uns parlent d'une parole magique - personne n'est contre. La fonction d'une idée directrice se situe dans sa capacité de focaliser les représentations sociales relatives à un développement réalisable ou souhaitable de manière symbolique et convaincante. Elle oriente les perceptions, les réflexions et les décisions à travers les contextes institutionnels différents et sur les niveaux divers de la concrétisation. Elle devait avoir juste la dose d'imprécision qu'il faut pour être adaptée par les acteurs sociaux dans leurs différents contextes et rationalités systémiques d'action. Ainsi, elle dispose d'une certaine potentialité pour initier des processus de compréhension réciproque et des coordinations d'action (Dierkes, HofFmanN \& Marz 1992).

Mais il faut bien s'en rendre compte: Parler d'un consensus sur le statut d'idée directrice ne veut pas dire accord sur son interprétation ni sa traduction dans des problèmes, chiffres et mesures concrètes. De telles définitions sont l'enjeu d'une vaste panoplie des politiques du discours qui s'opposent, et qui d'ailleurs se situent plus ou moins dans les courants polarisés du débat public sur l'environnement évoqués au début (modernisation technico-écologique/restructuration politico-écologique). On peut bien se disputer alors sur ce qui signfie la liberté ou la justice dans un cas concret. Il est même bien difficile de trouver un accord provisoire, révisable. Ainsi la dernière commission d'enquête de l'Assemblée Nationale (Deutscher Bundestag) n'a réussi à formuler un consensus ni sur l'état des choses ni sur les programmes d'action à aborder. Cela se voit dans les votes particuliers de quelques membres de la commission cachés dans le texte, mais d'importance générale, qui se réfèrent p.ex. à l'urgence et la dimension des mesures dans un champ d'action concret (DEuTSChER Bundestag 1998).

Les expériences sociales avec un peu trop d'espoir de planification et de contrôle de la part des dirigeants suggèrent d'interpréter cette idée directrice plutôt comme règle négative. Celle-ci indique ce qu'il faut éviter et ne fonctionnera pas comme état final et positif de la société. Le concept «développement durable» comprend aussi bien des chances que des risques pour les participants du discours. Les chances se situent dans sa per- 
spective intégrante vis-à-vis les dimensions économiques, écologiques et sociales des activités humaines dans le contexte mondial. Ainsi l'idée de durabilité encourage le remplacement d'une politique d'environnement à court terme, le «management de crise», qui vient après pour réduire les dégâts, par une stratégie d'action à long terme, qui rend compte de la complexité des relations intersystémiques et qui suit le principe de précaution - avec tous ses problèmes inhérents. Les protagonistes de la protection de l'environnement s'y réfèrent alors pour en finir avec l'opposition établie entre politique économique et politique écologique. Les risques liés à la notion se manifestent dans la possibilité de dresser les dimensions l'une contre l'autre et de créer ainsi des situations de blocage réciproque. Alors, les représentants de l'économie peuvent s'en servir pour repousser les revendications écologiques.

Comme ce débat sur la traduction de l'idée de développement durable dans des actions concrètes s'adapte à la structuration conflictuelle du discours public, le savoir scientifique ne peut pas vraiment arriver à suggérer une solution. Il faut bien voir que les expertises et contreexpertises économiques ou écologiques se perdent dans la critique réciproque. Les interprétations rivalisantes se fondent sur des régimes d'intérêts, de temps, de situations, de cultures et de connaissances très hétérogènes. L'idée de durabilité reste néanmoins créatrice de rencontre, de compréhension réciproque ne fût-ce que pour voir plus clair dans ce qui sépare les diverses positions. Est-ce plutôt du bavardage ou le début des innovations non seulement techniques mais aussi bien institutionelles, structurelles, culturelles et sociales? Cette question reste ouverte.

\section{Durabilité et société du risque}

\subsection{Société du risque}

Comment faut-il concevoir le rapport entre l'idée du développement durable et la «société du risque» (BECK 1986) ? La perspective sociologique caractérise les sociétés modernes du type ouest-européen ou d'Amérique du Nord par plusieurs principes et structures institutionels de base: la différenciation fonctionnelle; l'organisation de l'ensemble dans un cadre territorial et national; l'économie capitaliste, accompagnée par des dispositifs de sécurité sociale; la séparation entre travail salarié et travail reproductif; l'organisation des relations intimes dans la famille restreinte; la science comme première source publique de légitimation pour décisions collectives; le laïcisme et la privatisation de la croyance etc. Dans ces sociétés, il y a un consensus collectif d'arrière-fond sur la constitution de la réalité commune qui est basé sur la capacité interprétative de la science, l'efficacité de la technique et la pragmatique d'action quotidienne. Ce consensus remplace la religion (Religionsersatz), et il prend la place de la religion (Er- satzreligion): Le sociologue MAX WeBER écrivait en 1919 dans son essai sur «Le métier de scientifique» (Vom inneren Beruf zur Wissenschaft), qu'il ne fallait pas confondre le processus occidental de rationalisation avec une «connaissance générale et grandissante de toutes les conditions de la vie, qui nous dominent. Mais qu'il signifiera bien autre chose: Le savoir ou la croyance dans la possibilité d'une connaissance complète si on le voulait. (...) Qu'on pourrait, en principe maîtriser toutes les choses à travers le calcul» (WEBER 1968: 593). Ce n'est pas par hasard qu'il parle de croyance dans ce contexte.

Bien évidemment, les sociétés modernes sont dans tous leurs aspects, dans toutes les pratiques d'action, de la politique en passant par l'achat des légumes au marché jusqu'à la procréation des enfants, beaucoup plus imprégnées par les savoirs scientifiques que n'importe quelle société d'antan. Mais cette expansion des connaissances scientifiques n'a pas du tout conduit vers la clarté et la force des choses dans les décisions, qu'on attendait, bien au contraire: L'idée d'un agrandissement linéaire des connaissances scientifico-techniques et des maîtrises techniques des phénomènes naturels ou sociaux, ainsi que les promesses de progrès et de sécurité basées là-dessus, ont bien été bouleversées par le débat sur l'environnement et les risques dans les années 19701990. Les accidents de Harrisburg, Seveso ou Chernobyl ont souligné la normalité des catastrophes (PERROW 1988). Les taux limites pour les substances nuisantes fixent aussi bien des standards d'intoxication (BECK 1993). Les conflits sur l'effet de serre, le dioxine ou l'ozone montrent que les effets pervers des actions légitimes peuvent menacer les fondements de l'existence humaine. Les processus continus d'expansion des sciences et des techniques dans les sociétés modernes font augmenter les deux: les connaissances aussi bien que les non-connaissances; les possibilités d'intervention et les ambiguïtés; les sécurités et les insécurités. Le sociologue ULRICH BECK a proposé de parler d'une "société du risque», quand les conflits centraux d'une société portent sur les perceptions, les distributions, les victimes des risques produits par la société elle-même (BECK 1986). De tels risques sont aussi bien réels, discursifs et sociaux: dangers réels, résultats des processus d'interprétation, suites des actions humaines (LATOUR 1991: 15). Ils ne sont pas perceptibles à l'oeil nu, mais ils obligent la médiation du savoir par les experts scientifiques. Ils ne peuvent pas être exclus; on ne les connaît pas d'avance. Ils ne se limitent pas à un territoire local restreint. Ils ne s'adaptent pas à la logique sociale établie pour régler l'attribution de responsabilité causale ou morale parce qu'ils ne sont pas le résultat d'une intentionalité humaine, mais des effets pervers et/ou de cumulation (EwaLd 1991). À la différence des dangers traditionnels, de tels risques renvoient aux actions et décisions humaines qui les produisent. 


\subsection{Société des dangers}

Le terme diagnostic «société du risque», qui d'ailleurs ne se réduit pas aux phénomènes discutés dans cet article, mais comprend bien d'autres types de risques «sociaux» (pauvreté, exclusion etc. (BECK 1986)), est à la fois bien et mal choisi. Bien, parce qu'il a réussi à saisir une certaine ambiance collective, l'esprit du temps, dans une catégorie générale qui dépasse et comprend les secteurs différents de la société. Mal, parce que, dans un sens plus généralisé, les sociétés modernes ont été depuis toujours des «sociétés du risque» - c'est même leur forme normale d'existence. Qu'est-ce que cela veut dire ? La notion de 'risque' désigne un arrangement cognitif spécifique, une combinaison de calcul des probabilités avec le principe de certitude qui devient 'assurance' (Bonß 1995; BERNSTEIN 1997). Il s'agit là d'un produit historique né avec la société capitaliste et industrielle. Ce concept réduit les effets des contingences possibles du futur. Ainsi il permet d'agir dans le présent: on mise sur la réussite d'une action et les bénéfices qui en résultent. Contre le cas d'un échec possible et connu dans sa forme, on prend ses précautions - normalement sous la forme «classico-moderne» d'un contrat d'assurance. Dans ce sens, le risque fournit un modèle et une pratique pour s'arranger avec un futur incertain. Comme la planification ou l'incrémentalisme d'un «step by step», le concept social du risque est une modalité de la maîtrise sociale de la précarité, de l'incertitude, de la peur du futur, une pratique spécifique pour créer des certitudes pour agir dans le présent. Cette garantie se fonde sur la statistique et la solidarité contractuelle d'une communauté d'assurance.

Mais c'est justement cette fonction de domestication du futur qui commence à s'effacer si la science et la technique ne tiennent plus leurs promesses de sécurité. Alors les dangers - compris comme risques non plus calculables, mais risques arrivés - reviennent au centre de la société. Dans ce sens, les sociétés contemporaines sont des «sociétés des dangers». Elles retrouvent les risques qu'elles ont courus sous le signe des dangers produits par elles-mêmes (LAU 1989; LUHMANN 1991). Ainsi naissent des nouvelles constellations conflictuelles: entre les producteurs/profiteurs du risque et ceux touchés (éventuellement) par ses conséquences graves; entre les experts savants et les «ignorants»; mais aussi bien entre les experts qui promettent la sécurité, et les contre-experts qui mettent en garde contre les effets pervers et les aspects non maîtrisés.

\subsection{La modernisation réflexive}

La logique de la production des dangers indique l'ambivalence des processus sociaux de la modernisation: C'est justement leur «toujours plus» inhérent qui se retourne contre leurs promesses de sécurité et de prospérité. Ainsi, dans le processus occidental de rationalisation l'épisode de la société du risque et des dangers évoque plusieurs nouvelles expériences so- ciales collectives: l'ambiguïté du savoir scientifique et technique, les non-connaissances sur les effets pervers, la potentialité politique et la force mobilisatrice du nonsavoir. Les ambiguïtés apparaissent comme des incertitudes sur le plan cognitif, comme insécurités sur l'échelle sociale. Ainsi, cet épisode de la société moderne signale encore une autre ébranlement collectif: la perte de la certitude du futur. La transformation des risques (maîtrisés) en dangers (incalculables) est un processus d'insécurisation collective, qui bouleverse quelques fondements de base de la société moderne. La maîtrise du futur n'est plus une stratégie assurée; l'incertitude et l'insécurité reviennent à l'ordre du jour. UlRICH BECK parle dans ce contexte d'un processus de «modernisation réflexive» (BECK, GIDDENS \& LASH 1996). Cela ne veut pas dire réflexion consciente mais «revenir sur soi-même», «auto-confrontation»: les effets pervers de la modernisation simple, les dangers produits dans et par la société dénouent les bases de ce processus même. Bien sûr ils n'agissent pas directement sur le changement social. Ils ne le font que dans la mesure où ils sont communiqués par les acteurs sociaux, les discours, les conflits sur les savoirs, connaissances et normes, et ainsi deviennent des événements connus - ils en offrent d'ailleurs une vaste palette d'occasions. Dans ce sens, la modernisation réflexive suscite la réflexion. Dans une perspective d'utopie positive, il s'agit d'un processus d'apprentisage collectif qui produit un niveau de réflexion nouveau et qui élargit les horizons d'action. Les efforts consacrés au développement durable en fournissent peut-être un indice. Dans un prognostic négatif, il n'y a pas moyen de contrôler ni de canaliser les processus de modernisation.

\subsection{La politique des fictions de savoir}

Il se peut que le terme de durabilité soit le centre d'un nouveau méta-récit (dans le sens de LyOTARD), qui se distingue par une caractéristique centrale des anciens méta-récits de la modernité (comme le Marxisme, le Progrès): pendant que les derniers ont parlé des processus quasi-naturels et des lois de développement, le débat sur le développement durable souligne les capacités humaines et sociales d'action - il faut agir, sinon la catastrophe va arriver. Cette action ne vient pas de soi, du ciel, de la nature etc., mais d'une réalisation et mise en place collective, consciente qui résulte de la prolifération des effets pervers. La durabilité vise une nouvelle technologie de la maîtrise du futur, qui n'a pas encore trouvé sa concrétisation institutionnelle. Cette ouverture implique une certaine ambivalence. Jusqu'ici, le débat qui tourne autour la durabilité semble évoquer une nouvelle croyance dans les experts, la science, la technique. Là, il s'agit bien d'une forme de technocratie, fût-elle illuminée, qui court le risque de prolonger les principes de base de la modernisation tout court. Vont au moins dans ce sens l'instrumentalisation généralisée de l'idée de durabilité dans les sciences ainsi que les ten- 
tatives de fixer des entités mesurables et d'en tirer les conséquences et les urgences d'action. Du problème des risques on parle peu; quand c'est le cas, il devient déclencheur des conflits (Deutscher Bundestag 1998). Il y a bien une logique dans cet effet.

L'importance des risques devenus dangers se situe justement dans leur capacité d'attirer l'attention collective aux limites de la prévision scientifico-technique et de la précision du savoir scientifique. Ainsi, l'existence des conflits - fût-ce sur la revendication des connaissances (scientifiques) - apparaît comme «normalité» dans un nouveau sens: il ne s'agit plus des problèmes d'un savoir encore incomplet, mais des conflits qui lui sont inhérents et résultent systématiquement de la complexité de sa production. Devant cette situation, il ne faut ni envisager un stade non-conflictuel ni revenir sur le degré de scientificité des sociétés modernes. La catégorie du risque signale plutôt les limites de la production des connaissances unanimes. Néanmoins, dans le débat sur le développement durable, on réclame ou postule assez souvent l'unanimité d'un savoir quand il s'agit de légitimer une action ou une non-action. Le fait de savoir si - et sur quel point - les stratégies de durabilité sont le résultat d'un processus d'apprentisage collectif sera mesuré à leur capacité de promouvoir des modes institutionnels nouveaux de traiter les incertitudes produites. L'expérience montre qu'il y aura toujours des découvertes des risques/dangers inconnaissables d'avance. Et il y aura des évaluations divergentes de leur acceptabilité. Il est difficile d'en envisager un consensus définitif.

La théorie culturelle de Mary Douglas, Aaron WILDAVSKY ou MichaEL THOMPSON, malgré toute la critique qu'il faut lui adresser (Keller \& Poferl 1998), a bien montré les effets de la concurrence de différents schèmes culturels d'interprétation jusqu'au coeur de la construction du savoir sur les «faits» dans les sciences. La connaissance produite des faits de la réalité et de ses processus est bien sûr indispensable et ne doit pas être diluée dans un pluralisme de salon, sans conséquences. Mais il n'y aura pas non plus un savoir objectif à l'ancienne. Il faudra plutôt parler des fictions de savoir socialement objectivées à durée limitée. La vérité scientifique absolue ne fonctionnera plus comme ressource finale de la légitimation des actions. Le caractère systématiquement provisoire et l'ambiguïté de principe des connaissances scientifiques pourraient entrer dans l'espace public et ainsi devenir le fondement d'une décision sociale sur une justification suffisante, qui accordera une place importante à d'autres types de savoir et de valeur. Il y a plusieurs points d'ancrage d'une telle objectivation sociale (et non plus seulement scientifique) des fictions de savoir, p.e. la différenciation plus raffinée et nécessaire entre les risques et dangers écologiques divers, la rôle des expériences dans ce domaine (1), la médiation des conflits (2) et des dispositifs structurels (3).
(1) Même sans connaissance précise de toutes les conséquences potentielles d'une intervention dans les systèmes écologiques, économiques et sociaux les sociétés modernes disposent, en ce qui concerne les types de risque divers, d'un certain stock des connaissances par expérience sur leurs portées possibles. Ainsi, la commission d'enquête déjà mentionnée résume: Plus l'intervention va loin dans les systèmes, plus fort est l'impact concret; plus nombreux sont les cas d'intervention, plus des effets pervers se produisent dans le temps et dans l'espace. Suivant le principe de précaution, il faut donc se restreindre là où une évaluation des risques possibles indique des conséquences graves ou catastrophiques (même rares). Là il faut choisir, si possible, des approches prudentes, qui tolèrent les fautes, qui ne créent pas des faits accomplis mais révisables (Deutscher Bundestag 1998: 53).

(2) Ces dernières années les institutions politico-administratives ont organisé de plus en plus des forums de concertation pour accompagner ou permettre certaines de leurs décisions (p.e. des incinérateurs, sur la manipulation génétique des plantes). Un règlement spécial et précis pour les participants devait permettre une compréhension réciproque, même un consensus entre les positions opposées. Assez souvent les forums de médiation et les tables rondes se fondent sur les idées de JÜRGEN HabERMAS sur le discours égal, sans jeu de pouvoir. Mais actuellement, leur boom connaît une certaine désillusion: on peut constater plus d'échecs que de succès dans leur mise en pratique. Cela dépend du champ et du type de conflit où ils sont adoptés: Un résultat consensuel est possible, s'il s'agit d'un conflit d'interêt classique. On arrive encore à un consensus s'il est possible de transformer un conflit de risque dans un tel conflit classique. Mais dans la plupart des cas l'échec des concertations s'explique par le caractère inapproprié de leur rationalisme qui ne s'adapte pas à la solution des conflits de risques (Keller \& PoferL 1994). Ce diagnostic se fonde sur (au moins) trois raisons: Premièrement il y a une préstructuration des mesures de concertation par des rapports inégaux de connaissance. Deuxièment il n'est pas chose aisée de séparer (jugement de) fait et (jugement de) valeur. $T r$ oisièmement les conflits sur les risques semblent être au fond des conflits de valeur, sur les questions d'une vie bonne et juste. Ils réclament donc un débat qui n'est pas prévu par le biais rationaliste des dispositifs.

La cause finale de ce bilan déficitaire réside dans le fait qu'ils arrivent très (et peut-être trop) tard dans le processus de l'application d'une technologie, et non dans le processus de son développement ou même de discussion sur les «besoins» et les options possibles pour leur répondre. Ce qui domine c'est la pratique «end of pipe». Mais une technologie est une forme de la politique. L'agir professionnel des scientifiques, des ingénieurs, des techniciens, dans les institutions de promotion de la 
recherche et du développement est implicitement politique, parce que c'est là que les décisions riches d'implications et de conséquences sociales sont prises. Quand la nature et la technique se révèlent non plus comme existence et chose objective, neutre, mais comme constructions politiques et contingentes, elles perdent leur statut d'enclaves pré- ou a-consensuelles. Cette perte d'objectivité complique les possibilités de consensus basés sur la vérité des faits. Devient nécessaire une entente provisoire, révisable, sous réflexion permanente (LUHMANN 1995). L'ouverture discursive des processus du développement des techniques et leur enrichissement par des réflexions sur les valeurs pourra réduire leur auto-dynamique et dépendance immédiate du marché économique et élargir la gamme des scénarios et options alternatives, avant que le processus scientifico-technique et économique de clôture d'un champ d'action ne soit terminé (Keller \& HaJeR 1996).
(3) La concurrence sur les définitions du risque et la facticité des faits devient l'état normal des sociétés modernes et réflexives. Il faut trouver les moyens de le stabiliser institutionnellement, justement pour en tirer le profit sous forme de production des savoirs. Ainsi deviennent intéressants tous dispositifs institutionnels qui assurent le courage civique dans les domaines non publics de la production du savoir scientifico-technique, la fabrication et la promotion des connaissances du risque et de la critique. Un moyen sera la promotion et gratification institutionnelle des hypothèses sur les dangers; ou encore la mise en réseau systématique et interdisciplinaire des connaissances. Ce n'est ni possible ni souhaitable d'éviter tout risque; mais il semble possible de créer les conditions de production systématique des connaissances. La promesse simple de sécurité sera remplacée par la négociation discursive du choix et de la distribution des risques. L'adaption de l'idée de durabilité comme idée directrice du développement et de la mise en place des techniques, des produits ou dans la

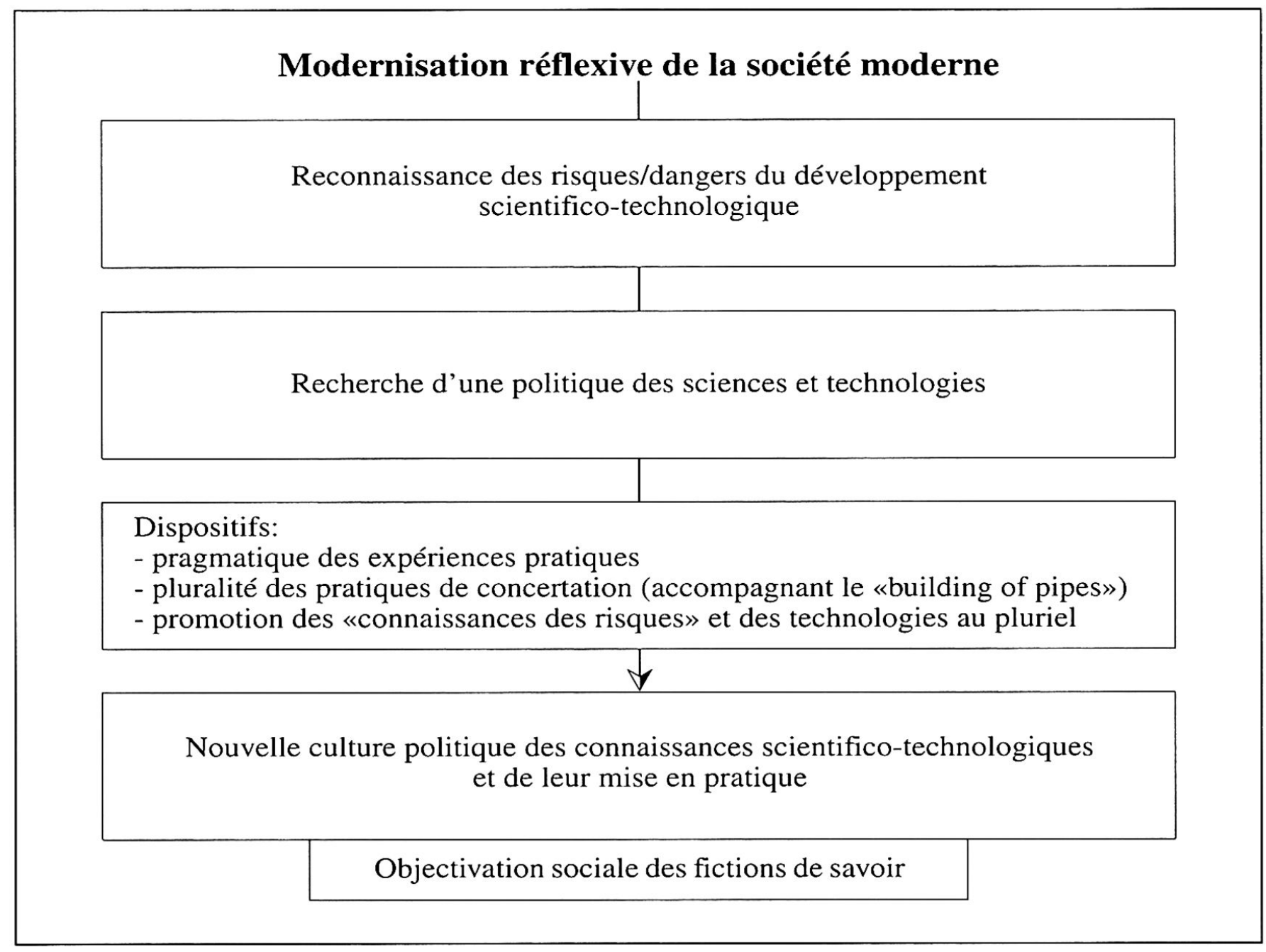

Tab. 2: Points d'ancrage d'une politique des «fictions de savoir» 
formation universitaire des ingénieurs et techniciens en fournit des occasions, si elle est consciente de la thématique des risques. Cependant, tout cela va contre la tendance forte de la dynamique économique qui pousse les savoirs scientifiques et les techniques vers le marché et l'utilité économique. Le temps entre production des connaissances, développement des technologies et adaption sur le marché se raccourcit de plus en plus dans l'ère des «sciences based industries».

\section{Conclusion}

Le développement durable est un concept de transformation des dimensions pratiques et symboliques des rapports sociaux à la nature qui est socialement et historiquement ouvert. Cette transformation ne peut pas se fonder sur la dynamique propre des processus de modernisation ou sur une tendance évolutionniste, parce que les institutions de la société moderne sont «aveugles» vis-à-vis de leurs effets pervers. Les capacités de modelage du développement des sociétés modernes dépendent des rapports de force et de conflit, des nécessités d'action perçues, des possibilités et buts d'action des acteurs sociaux. La perception des problèmes et l'évaluation des potentialités d'action ne sont pas que des questions de simple bonne volonté, mais le résultat des structures d'opportunité sociales (et écologiques), résultat de la politique im- ou explicite des modes de vie. Il s'agit de la transformation des pratiques sociales, des arrangements institutionnels et des orientations normatives de la société moderne sans pertes des avantages de la modernité (JAHN \& STIESS 1997: 32).

L'idée de durabilité ne brise pas les promesses de la modernité: améliorer les conditions de vie par des processus techniques, économiques et sociaux, à la base des connaissances scientifiques. C'est une réaction contre les effets pervers qui naissent dans la modernisation tout court. Ainsi elle peut devenir le cas exemplaire d'une modernisation réflexive. Sont envisagées trois corrections de l'ancienne dynamique de modernisation: l'autorestriction de l'exploitation sociale de la nature en fonction des conditions d'existence des générations futures; l'autorestriction de l'exploitation nationale de la nature en fonction d'une justice mondiale du développement; l'autorestriction des dynamiques et rationalités propres aux divers sous-systèmes de la société moderne en fonction de l'interconnexion des aspects écologiques et sociaux (BRAND 1997: 14). Cela implique une restructuration des terrains établis de conflit dont les luttes de définition viennent juste de commencer. Leurs conséquences institutionnelles - surtout en ce qui concerne les risques - sont encore dans le brouillard. La définition scientifique des potentialités de risque et des limites écologiques, économiques et sociales sera toujours contestée. Elle ne peut pas légitimer la définition normative des buts d'action. La société du risque et des dangers évoque une nouvelle mise en relation, un nouveau rapport entre science (savoir) et politique qui remplacera celui de la modernisation ancienne, devenu tradition, naturel.

\section{Bibliographie}

BECK, U. (1986): Risikogesellschaft. - Frankfurt/Main: Suhrkamp.

BECK, U. (1993): Politische Wissenstheorie der Risikogesellschaft. - In: BECHMANN, G. [ed.]: Risiko und Gesellschaft. - Opladen: Westdeutscher Verlag: 305326.

Beck, U., Giddens, A. \& S. LaSh (1996): Reflexive Modernisierung. - Frankfurt/Main: Suhrkamp.

Bernstein, P.L. (1997): Wider die Götter. - München: Gerling.

Bonß, W. (1995): Vom Risiko. - Hamburg: Hamburger Edition.

BRand, K.-W. (1997): Probleme und Potentiale einer Neubestimmung des Projekts der Moderne unter dem Leitbild «Nachhaltige Entwicklung». - In: BRAND, K.W. [ed.]: Nachhaltige Entwicklung. - Opladen: Leske \& Budrich: 9-34.

Brand, K.-W., Eder, K. \& A. Poferl (1997): Ökologische Kommunikation in Deutschland. - Opladen: Westdeutscher Verlag.

Bund/Misereor [ed.] (1996): Zukunftsfähiges Deutschland. - Basel: Birkhäuser.

Dierkes, M., Hoffmann, U. \& L. Marz (1992): Leitbild und Technik. - Berlin: Sigma.

Deutscher Bundestag [ed.] (1998): Konzept Nachhaltigkeit. Abschlußbericht der Enquete-Kommission «Schutz des Menschen und der Umwelt». - Bonn.

Ewald, F. (1991): Der Vorsorgestaat. - FrankfurtMain: Suhrkamp.

Hesse, M. (1997): Alter Wein in neuen Schläuchen. Stadtenwicklung im Licht der Agenda 21. - In: Politische Ökologie, 52: 38-41.

JAHN, T. \& I. STIESS (1997): Nachhaltigkeit. Eine Herausforderung an die Sozialwissenschaften. - In: Wechselwirkung, 87: 30-35.

Jonas, H. (1990): Le principe de responsabilité. Une éthique pour la civilisation technologique. - Paris: Cerf. Keller, R. (1995): Medienselektivität und Umweltberichterstattung in der Bundesrepublik Deutschland. = MPS-Texte 1, München: MPS.

Keller, R. (1998): Müll - Die gesellschaftliche Konstruktion des Wertvollen. - Opladen: Westdeutscher Verlag.

Keller, R. \& M. Hajer (1996): Modernisierung im Gespräch. - In: Wechselwirkung, 77: 56-61.

Keller, R. \& A. Poferl (1994): Habermas und der Müll. - In: Wechselwirkung, 68: 34-40.

Keller, R. \& A. Poferl (1998): Vergesellschaftete Natur: Öffentliche Diskurse und soziale Strukturierung. - In: BRAND, K.-W. [ed.]: Soziologie und Natur. - Op- 
laden, Westdeutscher Verlag: 117-144.

KunN, S. \& B. KurTe (1997): Kommunale Herausforderung. - In: Politische Ökologie, 52: 42-45.

LAU, C. (1989): Risikodiskurse. - In: Soziale Welt, 3: 418-436.

LATOUR, B. (1991): Nous n'avons jamais été modernes. - Paris: La Découverte.

Luhmann, N. (1991): Soziologie des Risikos. - Berlin: De Gruyter.

LuhmanN, N. (1995): Über Natur. - In: LuhmanN, N.: Gesellschaftsstruktur und Semantik IV, Frankfurt/ Main: Suhrkamp: 9-30.

Perrow, C. (1988): Normale Katastrophen. - Frankfurt/Main: Campus.

SACHVERSTÄNDIGENRAT FÜR UMWELTFRAGEN (1996): Umweltgutachten 1996. Zur Umsetzung einer dauerhaft-umweltgerechten Entwicklung. - Stuttgart: Metzler.

Weber, M. (1968): Gesammelte Aufsätze zur Wissenschaftslehre. - Tübingen: Mohr.

\section{Résumé: Le développement durable}

\section{dans la société du risque: le cas allemand}

L'auteur discute le rapport entre le débat allemand sur le Développement Durable et le concept sociologique d'une «société du risque». La discussion allemande sur le développement durable a tendance à ignorer les questions du risque. Ainsi, elle risque de ne faire que prolonger les dynamiques bien connues de la modernisation traditionnelle. La première partie du texte dresse un bilan critique du débat sur la durabilité à l'échelle nationale et locale. L'idée de durabilité est une idée régulatrice et directrice qui comprend aussi bien des chances que des problèmes. Les concepts sociologiques d'une société du risque (qui signifie «société des dangers») et de la modernisation réflexive indiquent que le savoir scientifique a tendance à perdre l'unanimité qui lui fut attribuée dans la société moderne. Il s'agit là d'un point central pour la base des connaissances scientifiques en matière de stratégies de durabilité. Cet argument est développé à travers la discussion de trois points d'ancrage possible d'une «politique des fictions de savoir», c'est-à-dire des dispositifs qui permettraient à la société de s'arranger avec l'incertitude des connaissances scientifiques.

\section{Zusammenfassung: Nachhaltige Entwicklung in der Risikogesellschaft: Der Fall Deutschland}

[Die deutsche Fassung ist beim Autor erhältlich.]

Der Beitrag diskutiert das Verhältnis der deutschen Diskussion über Nachhaltige Entwicklung zum sozialwissenschaftlichen Begriff der «Risikogesellschaft». Dazu wird die These formuliert, dass die Nachhaltigkeitsdebatte Risikofragen tendenziell vernachlässigt und so Gefahr läuft, die Dynamiken herkömmlicher Modernisierungsprozesse fortzuschreiben. In einem ersten Teil wird eine kritische Bilanz der Nachhaltigkeitsdiskussion auf nationaler und lokaler Ebene gezogen. Nachhaltigkeit kann als regulative Idee oder gesellschaftliches Leitbild verstanden werden, mit dem Probleme und Chancen verbunden sind. Die sozialwissenschaftlichen Konzepte der Risiko- oder Gefahrengesellschaft sowie der reflexiven Modernisierung weisen darauf hin, dass wissenschaftliches Wissen zunehmend seine ursprünglich zugeschriebene Eindeutigkeit verliert. Dies ist von zentraler Bedeutung für die Wissensbasis von Nachhaltigkeitsstrategien. Abschliessend wird deswegen an drei Ansatzpunkten diskutiert, wie eine «Politik der Wissensfiktionen» ansetzen kann, d.h. wie ein gesellschaftlicher Umgang mit unsicheren Wissensbeständen vorstellbar ist.

\section{Summary: Sustainable Development in the Risk Society: the German Case}

This paper discusses the relations between the German discussion on Sustainable Development and the social sciences concept of «risk society». It is argued that the sustainability debate shows tendencies to ignore risk issues and runs the danger of encouraging the dynamics of traditional modernisation. The argument starts with a critical review of the German sustainability debate at the national and local levels. Sustainability can be understood as a regulatory idea which involves both problems and opportunities. The concepts of risk society, society of dangers and reflexive modernisation indicate that scientific knowledge is losing its originally attributed clearness. This is a very important point for the know-ledge base of sustainability strategies which is taken up in the final part. This discusses three possible entries for a «politics of knowledge fiction», that is to say the question of how to organise social handling of uncertain knowledge.

Dr. Reiner Keller, Lehrstuhl für Soziologie, WISO-Fakultät, Universität Augsburg, D-86135 Augsburg.

e-mail: reiner.keller@wiso.uni-augsburg.de

\section{Manuskripteingang/received/rentrée du manuscrit: 5. Januar 1999 \\ Annahme zum Druck/Accepted for publication/ acceptation à l'impression: 4. August 1999}

